ARTICLE

\title{
Structural insight in the toppling mechanism of an energy-coupling factor transporter
}

\author{
Lotteke J.Y.M. Swier ${ }^{1,2}$, Albert Guskov ${ }^{1,2} \&$ Dirk J. Slotboom ${ }^{1,2}$
}

Energy-coupling factor (ECF) transporters mediate uptake of micronutrients in prokaryotes. The transporters consist of an S-component that binds the transported substrate and an ECF module (EcfAA'T) that binds and hydrolyses ATP. The mechanism of transport is poorly understood but presumably involves an unusual step in which the membrane-embedded S-component topples over to carry the substrate across the membrane. In many ECF transporters, the S-component dissociates from the ECF module after transport. Subsequently, substrate-bound S-components out-compete the empty proteins for re-binding to the ECF module in a new round of transport. Here we present crystal structures of the folate-specific transporter ECF-FolT from Lactobacillus delbrueckii. Interaction of the ECF module with FolT stabilizes the toppled state, and simultaneously destroys the high-affinity folate-binding site, allowing substrate release into the cytosol. We hypothesize that differences in the kinetics of toppling can explain how substrate-loaded FolT out-competes apo-FolT for association with the ECF module.

\footnotetext{
${ }^{1}$ Groningen Biomolecular Science and Biotechnology Institute, University of Groningen, Nijenborgh 4, 9747 AG Groningen, The Netherlands. 2 Zernike Institute for Advanced Materials, University of Groningen, Nijenborgh 4, 9747 AG Groningen, The Netherlands. Correspondence and requests for materials should be addressed to A.G. (email: a.guskov@rug.nl) or to D.J.S. (email: d.j.slotboom@rug.nl).
} 
E nergy-coupling factor (ECF) transporters are a recently discovered class of ATP-binding cassette (ABC) transporters ${ }^{1-4}$. The transporters are exclusively present in prokaryotes, where they catalyse the uptake of a wide variety of micronutrients, typically water-soluble vitamins and transition metals such as $\mathrm{Ni}^{2+}$ and $\mathrm{Co}^{2+}$ (ref. 3). ECF transporters consist of two identical or highly similar cytoplasmic ATPases, EcfA and

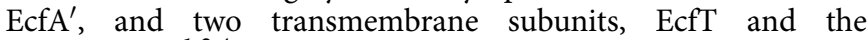
S-component ${ }^{1,2,4}$. In contrast to classical $\mathrm{ABC}$ importers ECF transporters do not make use of additional substrate-binding domains or proteins. The membrane-embedded S-component serves as both the substrate-binding and the substratetranslocation domain. The other three subunits (EcfA, EcfA' and EcfT) together form the ECF module that energizes substrate transport by the S-component ${ }^{4-6}$.

Based on the genomic location of the genes encoding the transporter subunits, ECF transporters have been categorized into two groups. Group I consists of dedicated transporters, in which the ECF module interacts with a single S-component, whereas group II ECF transporters share their ECF module with multiple S-components of different substrate specificities ${ }^{2}$. S-components from group II transporters can be released from the ECF module, and compete with each other for binding to the module ${ }^{7-9}$.

Several crystal structures of solitary S-components from a variety of organisms have been solved ${ }^{10-14}$. Despite little sequence identity $(\sim 15 \%)$ the S-components for different substrates share a similar fold with a core of six membraneembedded $\alpha$-helices. In addition, three crystal structures of complete group II ECF transporters from Lactobacillus brevis (L. brevis) have been solved, all containing the same ECF module and a different S-component: ECF-FolT, ECF-HmpT and ECF$\mathrm{PanT}^{5,6,15}$. The structures have provided the first glimpse of the mechanism of transport and the shared utilization of the ECF module by group II transporters.

Based on the structures, as well as mutational, accessibility and cross-linking studies on EcfT and S-components from different organisms ${ }^{4,14-16}$, a toppling mechanism of transport has been proposed, in which the S-component rotates in the lipid bilayer to expose its binding site alternately to the exterior (helices oriented as membrane-spanning) or the interior (toppled orientation with helices parallel to the membrane). The latter state was captured in the structures of the full complexes and is referred to as posttranslocation state ${ }^{15}$, in which the substrate has been released into the cytosol. ATP binding in the ATPase subunits is thought to cause a conformational change in EcfT, which allows the S-component to topple back to the outward facing state. In group II ECF transporters ATP binding and hydrolysis also leads to release of the substrate-free S-component from the ECF module and allows for competition among different substrate-bound S-components for the same ECF module $^{7-9}$.

Here we report crystal structures of the group II ECF transporter for folate from Lactobacillus delbrueckii ( $L$. delbrueckii) in the apo-state and in complex with the slowly hydrolysable ATP analogue AMP-PNP. In addition, we present a structure of solitary FolT from the same organism in the folatebound state. Comparison of the structures of FolT in the complex and in isolation reveals how solitary FolT captures substrate from the extracellular side, and how association with the ECF module is coupled to substrate release in the cytosol. We re-evaluate existing data in the light of new structures and provide a working model for the transport mechanism that can explain a crucial observation that led to the discovery of ECF transporters: the more effective competition of S-components in the substratebound state for the ECF module than proteins in the substratefree state.

\section{Results}

S-components for folate in L. delbrueckii. The genome of $L$. delbrueckii encodes two FolT homologues, which we named FolT1 and FolT2. They share 93\% sequence identity and have likely arisen from a recent gene duplication. FolT1 and FolT2 share only between 30 and $33 \%$ identity with the characterized FolT proteins from L. brevis, Enterococcus faecalis (E. faecalis) and Lactobacillus casei (L. casei) (Supplementary Fig. 1) $)^{5,14,17}$. Purified FolT1 and FolT2 in detergent solution bound folate with high affinity, with dissociation constants of $1.0 \pm 0.24$ and $3.1 \pm 1.4 \mathrm{nM}$, respectively (s.d.'s from three independent experiments, Supplementary Fig. 2). The affinities of FolT1 and FolT2 for folate are higher than the affinities determined previously for the FolT proteins from E. faecalis $(29.8 \mathrm{nM})^{14}$ and $L$. casei $(9 \mathrm{nM})^{17}$. FolT1 and FolT2 differ in only 12 residues, none of which interact with folate, with the possible exception of residue 122 (see below), which is a tyrosine in FolT1 and an asparagine in FolT2. The FolT1 mutant Y122N binds folate with a dissociation constant of $2.5 \pm 1.2 \mathrm{nM}$ (Supplementary Fig. 3), indicating that the tyrosine contributes to a slightly higher affinity, although the differences are small.

Crystal structure of folate-bound FolT1. We determined the crystal structure of folate-bound FolT1 at $3.0 \AA$ resolution (Table 1). FolT1 consists of six membrane-embedded $\alpha$-helices (Fig. 1a,b), which have a similar structure as other solitary S-components with specificities for various substrates ( $\mathrm{RibU}^{10}$, ThiT $^{11}$, BioY $^{12}$, NikM2 (ref. 13), FolT ${ }^{14}$ ). Structurally, FolT1 is most closely related to FolT from E. faecalis with which it shares $30 \%$ sequence identity.

Based on the hydrophobicity of the helical segments and the positive-inside rule ${ }^{18}$ solitary FolT1 is predicted to be oriented in the membrane with the $\mathrm{N}$ - and $\mathrm{C}$-terminal ends and loops $\mathrm{L} 2$ and L4 located in the cytoplasm, and loops L1, L3 and L5 facing the extracellular environment (Fig. 1b). However, deduction of the membrane orientation from the structure is difficult in this case because we know that the FolT protein can topple over in the context of the complete ECF transporter (see below). Nonetheless, it is plausible that solitary FolT can adopt the orientation shown in Fig. 1a as it is in agreement with molecular dynamics simulations that revealed a similar orientation for the solitary S-component ThiT in a lipid bilayer ${ }^{19}$.

Well-defined non-protein electron density inside the $\alpha$-helical core was assigned to the folate molecule (Supplementary Fig. 4). The occluded folate-binding site is lined by all six membrane $\alpha$-helices and is located close to the extracellular side of the membrane (orientation as in Fig. 1a), where it is shielded from the exterior by the L1 loop connecting helices 1 and 2, and the L3 loop between helices 3 and 4 . The substrate-binding pocket has a volume of about $1,400 \AA^{3}$, which leaves a vast amount of space for extensions on the folate molecule such as a polyglutamate tail. A detailed description of the interactions is provided in the supplement, but it is important to note that loop L1 and loop L3 provide many of the interactions with the folate molecule. The pterin moiety of the folate molecule forms a network of strong interactions with residues from helices 3 and 4 , and the connecting loop L3 (Supplementary Fig. 4c), while the aminobenzoate and glutamate moieties interact with residues from helices 5 and 6, and loop L1 (Supplementary Fig. 4d).

Transport activity of ECF-FolT1 and ECF-FolT2. FolT1 and FolT2 interact with the same ECF module to assemble into full ECF transporters. We purified the ECF-FolT1 and ECF-FolT2 complexes, reconstituted them into proteoliposomes and performed transport assays with radiolabelled folate (ECF-FolT2: 
Table 1 | Data collection, phasing and refinement statistics.

FolT1

\begin{tabular}{lc}
\hline Data collection & $C 121$ \\
Space group & \\
Cell dimensions & $108.91,77.54,89.45$ \\
$a, b, c(\AA)$ & $90.0,116.36,90.0$ \\
$\alpha, \beta, \gamma\left({ }^{\circ}\right)$ & $43.0-3.01(3.32-3.01)^{\star}$ \\
Resolution $(\AA)$ & $0.12(0.38)^{\star}$ \\
$R_{\text {merge }}$ & $5.3(2.18)^{\star}$ \\
$l / \sigma l$ & $82.3(44)^{\star}$ \\
Completeness (\%) & $2(1.8)^{\star}$ \\
Redundancy &
\end{tabular}

Refinement

Resolution $(\AA)$

No. of reflections

$R_{\text {work }} / R_{\text {free }}$

No. of atoms

Protein

Ligand/ion

Water

$B$-factors

Protein

Ligand/ion

Water

R.m.s. deviations

Bond lengths $(\AA)$

Bond angles $\left({ }^{\circ}\right)$

3.01
10,960
$0.235 / 0.286$
2,456
2,392
64
-
36
17
-
0.011
1.429

AMP-PNP bound ECF-FolT2

P1

$90.07,97.26,105.45$

$84.67,64.78,62.59$

$43.9-3.30(3.40-3.30)^{*}$

$0.026(0.86)^{\star}$

$12(1.10)^{\star}$

$94.4(78.8)^{*}$

$1.8(1.7)^{\star}$

3.30
33,723
$0.252 / 0.294$
15,554
15,430
124
-
159
158
-
0.006
1.404

apo ECF-FolT2

P1

$88.82,95.32,107.57$

$83.45,65.75,61.99$

$49.0-3.00(3.07-3.00)^{*}$

$0.04(0.92)^{\star}$

$10.5(1.65)^{\star}$

$92.6(86.6)^{x}$

$1.8(1.72)^{\star}$
3.00

41,549

$0.232 / 0.279$

15,398

15,398

For each structure, one crystal was used for data collection.

*Values in parentheses are for the highest-resolution shell.

Fig. 2a, ECF-FolT1: Supplementary Fig. 5). Both ECF transporters mediated association of radiolabelled folate with the proteoliposomes in the presence of luminal MgATP, but not in the presence of MgADP. Although these experiments suggest that the ECF transporters translocate folate across the membrane into the lumen, they are not conclusive. The reason for the ambiguity is that there are apparently more protein molecules present in the liposomes than folate molecules associating with them. Therefore it cannot be excluded that MgATP-dependent binding of folate to the proteins might have occurred, without translocation. In the case of ECF-FolT2 there was an almost seven-fold excess of protein complexes $(8.7 \mathrm{pmol}$, assuming a reconstitution efficiency of $100 \%)$ over folate molecules associated with the liposomes (1.3 pmol). Transport assays reported previously using other purified and reconstituted ECF complexes from Lactococcus lactis (L. lactis) and L. brevis, showed similar ambiguities, although they were not discussed. In these cases, we calculated protein to substrate excesses of 12.7-fold $\left(\mathrm{ECF}-\mathrm{NiaX}^{20}\right), 29.0$-fold $\left(\mathrm{ECF}-\mathrm{RibU}^{20}\right)$ and 627 -fold (ECF-FolT $\left.{ }^{5}\right)$.

To unambiguously show that ECF-FolT2 could transport folate across the membrane, we made use of the observation that the orientation of membrane proteins in liposomes is often scrambled during the reconstitution procedure: liposomes contain both right-side-out- and inside-out-oriented protein complexes. If the ECF complexes can catalyse transport rather than just binding, the inside-out-oriented complexes should allow export of luminal folate from the proteoliposomes in the presence of external MgATP. To test this prediction, we performed a folate uptake experiment as described above until a steady-state level of folate association was reached (after $16 \mathrm{~min}$, Fig. 2b), and then added external MgATP or MgADP to the liposomes. Efflux of the radiolabelled folate occurred on addition of $\mathrm{MgATP}$, but not on addition of MgADP, which shows that ECF transporters indeed translocate their substrates across the membrane and use an a

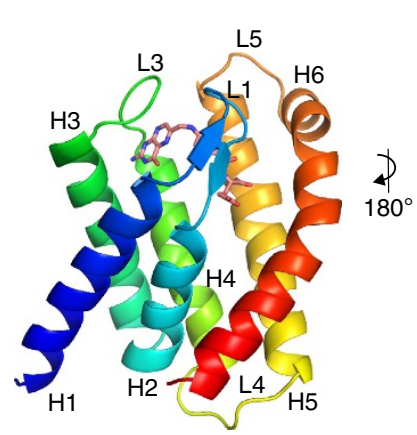

b

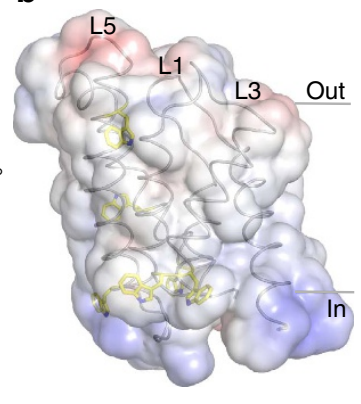

Figure 1 | Crystal structure of folate-bound FolT1. (a) Cartoon representation of FolT1 coloured from blue ( $\mathrm{N}$ terminus) to red ( $\mathrm{C}$ terminus), with folate shown in stick representation. H1-6 indicates $\alpha$-helices 1-6. The carbon atoms of folate are shown in deep salmon, the oxygen and nitrogen atoms are shown in red and blue, respectively. The colour coding for oxygen and nitrogen atoms remained throughout the article. The membrane orientation was derived from the hydrophobicity of the surface, the positive inside rule and the location of aromatic residues shown in $\mathbf{b}$, in which the transparent surface is coloured according to the surface electrostatic potential (negative potential in red and positive potential in blue), and tryptophan residues are shown in sticks with their carbon atoms coloured yellow. The bottom of the protein faces the cytoplasm, while the top faces the extracellular side of the membrane.

ATP-dependent mechanism. As a further control, we added a 1000 -fold excess of non-radiolabelled folate to the proteoliposomes after the steady-state level of folate association had been reached. The excess folate did not chase off the accumulated radiolabel, which again is consistent with transport rather than binding. 
To test whether ATP binding is sufficient for transport or ATP hydrolysis is required, we filled the proteoliposomes with ATP and EDTA (in the absence of $\mathrm{Mg}^{2+}$ ) or with the slowly hydrolysable ATP analogue AMP-PNP (in the presence of $\mathrm{Mg}^{2}{ }^{+}$) (Fig. 2a). In neither condition folate transport occurred, indicating that ECF-FolT mediates folate translocation only under ATP hydrolysing conditions.

Beside folate transport, we also measured the ATPase activity of ECF-FolT2 in the proteoliposomes, both in the presence and absence of $100 \mathrm{nM}$ of folate (Fig. 2c). The presence of folate did not significantly affect ATPase activity indicating poor coupling between transport and nucleotide hydrolysis. High futile ATPases activity has also been observed for the group I transporter BioMNY ${ }^{16}$. Comparison of the rate of ATP hydrolysis with the rate of folate transport corroborates that there is poor coupling, as the ATPase rate is more than four orders of magnitude higher than the transport rate. Apparently futile ATP hydrolysis is an inherent characteristic of the ECF transporter.

Crystal structures of ECF-FolT2 complexes. We determined crystal structures of the ECF-FolT2 complex from L. delbrueckii

a
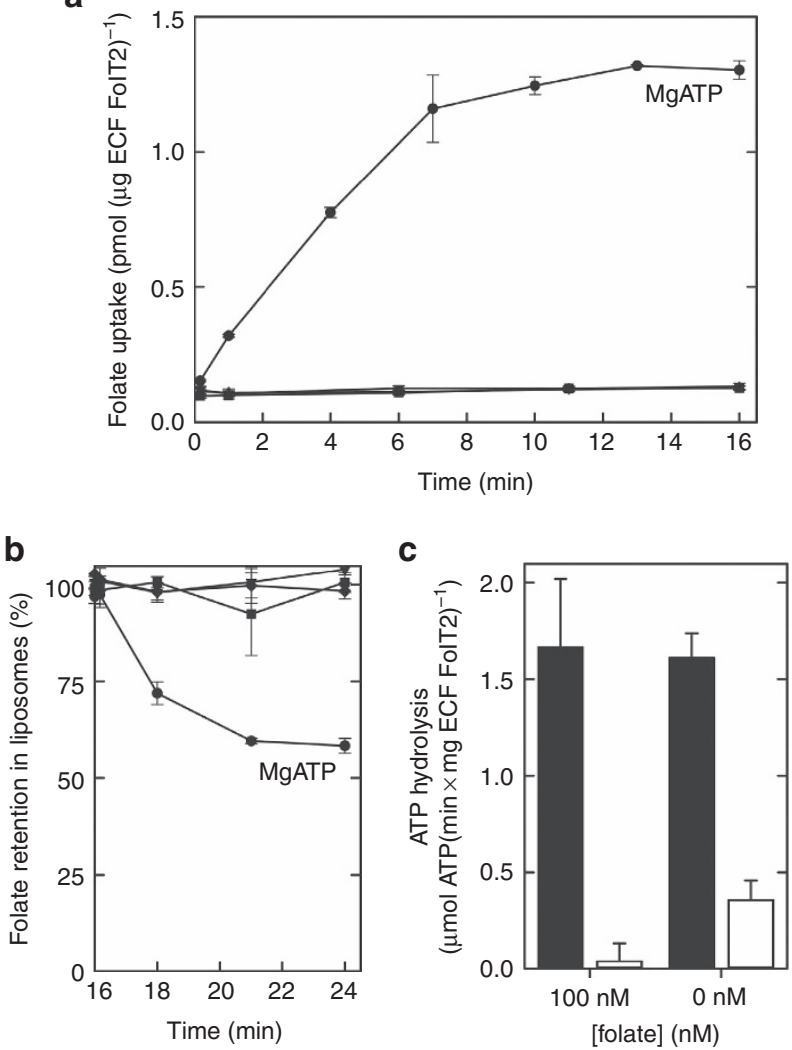

Figure 2 | Transport and ATPase activity of ECF-FolT2. (a) Transport activity of ECF-FolT2 in proteoliposomes loaded with $5 \mathrm{mM}$ of MgATP (circles), $5 \mathrm{mM} \mathrm{MgADP}$ (inverted triangles), $5 \mathrm{mM}$ MgAMP-PNP (triangles) or $5 \mathrm{mM} \mathrm{Na}{ }_{2}$ ATP plus $5 \mathrm{mM}$ EDTA (squares). (b) Efflux activity of ECF-FolT2 from proteoliposomes loaded with $5 \mathrm{mM}$ of MgATP, after accumulation of radiolabelled folate for $16 \mathrm{~min}$. At $t=16 \mathrm{~min}, 5 \mathrm{mM}$ of MgATP (circles), $5 \mathrm{mM} \mathrm{MgADP} \mathrm{(inverted} \mathrm{triangles),} 5 \mathrm{mM} \mathrm{Na}{ }_{2}$ ATP plus $5 \mathrm{mM}$ EDTA (squares) or $100 \mu \mathrm{M}$ non-radiolabelled folate (diamonds) was added to the reactions. (c) ATPase activity of ECF-FolT2 reconstituted in proteoliposomes (black bars) and background ATPase activity by empty liposomes (white bars). When indicated, folate was present both in the lumen of the liposomes and in the environment. The error bars show the s.d.'s from three independent measurements. in the apo state (no nucleotides or folate) and in an AMP-PNPbound state at 3.0 and $3.3 \AA$ resolution, respectively (Table 1). The overall structures of the two complexes are shown in Fig. 3 and Supplementary Fig. 6. Although both structures globally resemble the structures of the ECF transporters from $L$. brevis ${ }^{5,6,15}$, the corresponding subunits share only between 30 and $50 \%$ sequence identity. Importantly, the comparison of the structures of solitary FolT1 and the full ECF-FolT2 from a single organism now allows insight into the conformational transitions that take place during transport that cannot be obtained from the comparison of proteins with low sequence similarity from different organisms.

In both structures of ECF-FolT2, the ATPases EcfA and EcfA' are in an open conformation with the subunits separated thus leaving the two ATP hydrolysis sites incomplete. In the AMPPNP bound structure, EcfA and EcfA' each bind a molecule of the nucleotide, but binding does not lead to closure of the ATPase dimer (see below). EcfT can be divided into two domains: the transmembrane domain formed by five transmembrane helices (TMH1-5), and the coupling domain formed by the three coupling helices (CH1-3). The cytosolic side (bottom) of the coupling domain interacts with the ATPase subunits, whereas the membrane side (top) binds to the S-component. The membrane domains of EcfT are in different conformations in the two structures (see below). Compared with the putative membrane orientation of solitary FolT1 (Fig. 1a), FolT2 has toppled over and the helices lie roughly parallel to the membrane plane (Fig. 3a,c). Because the AMP-PNP and apo complexes have similar structures, we will focus our further discussion on the AMPPNP-bound structure, and consider the apo complex only when it differs from the AMP-PNP structure.

Comparison of solitary FolT1 with FolT2 in the complexes. The FolT2 proteins in the AMP-PNP-bound and apo complexes do not have folate bound. Nonetheless, the structure of substratefree FolT2 is very similar to the structure of solitary, folate-bound FolT1 with root mean squared deviation values of $1.35 \AA$ (FolT1 compared with FolT2 from the AMP-PNP-bound structure) and $1.25 \AA$ (FolT1 compared with FolT2 from the apo structure). All $\alpha$-helical segments and cytoplasmic loops are in almost identical conformations. The only differences are the conformations of the L1, L3 and L5 loops (Fig. 4a). In FolT1, L1 and L3 cover the folate-binding pocket and provide multiple interactions with the substrate, whereas they have moved away in the substrate-free state, creating a wide opening. On the movements of the L1 and L3 loops, the interactions with folate are lost and the high-affinity binding site is destroyed (Fig. $4 \mathrm{~b}$ discussed below). Loop L5 also adopts a slightly different conformation but this displacement does not affect the folate-binding site directly (Fig. 4a). The loss of the high-affinity binding site for folate in the complex explains why no electron density for folate was found in the structure of the AMP-PNP-bound complex even though a high concentration of the substrate was added during crystallization. Consistently, biochemical characterization of ECF-RibU has shown that riboflavin could bind only to solitary RibU, but not to the full complex $^{8}$. The structures also explain how folate is released into the cytoplasm, because the L1 and L3 loops that occluded the binding site in the solitary S-component are now moved and allow passage of the substrate towards the cytoplasm.

The structural resemblance of FolT1 and FolT2 indicates that only small conformational changes take place in S-component on substrate binding or release. This notion is consistent with EPR and pre-steady-state fluorescence experiments on the thiamin binding to the $\mathrm{S}$-component $\mathrm{ThiT}^{19}$ that showed minor conformational changes on binding and very rapid association 
a

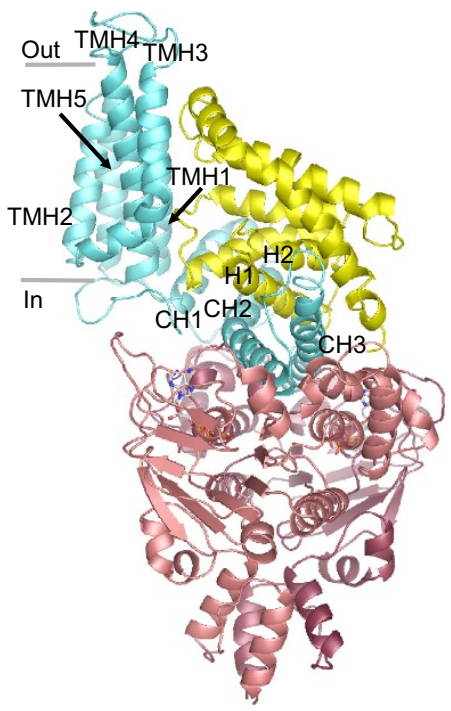

b
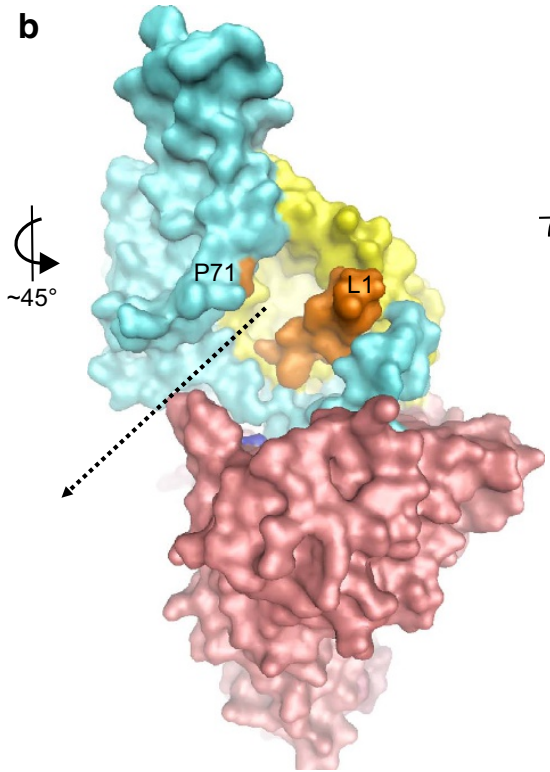

C

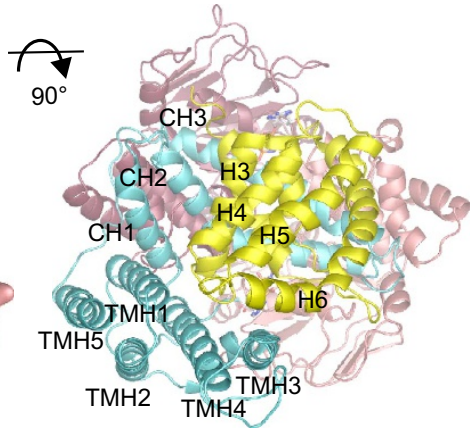

Figure 3 | Crystal structure of AMP-PNP bound ECF-FolT2. (a) Cartoon representation of ECF-FolT2 viewed from the plane of the membrane, with EcfA and Ecf $\mathrm{A}^{\prime}$ coloured in two shades of red, EcfT in cyan and FolT2 in yellow. AMP-PNP molecules are shown in sticks representation, with the carbon and phosphor atoms coloured grey and orange, respectively. The transmembrane helices of EcfT are indicated by TMH1-5 and the three coupling helices by $\mathrm{CH1}$-3. (b) Surface representation using the subunit colours from a. Loop L1 of FolT2 and proline 71 (P71) in TMH3 of EcfT are coloured orange. The pathway leading from the open folate-binding cavity to the cytosol is indicated by the dashed arrow. (c) View along an axis perpendicular to the membrane.

a

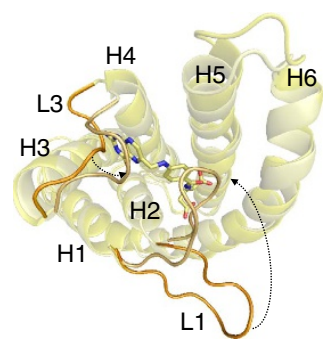

d

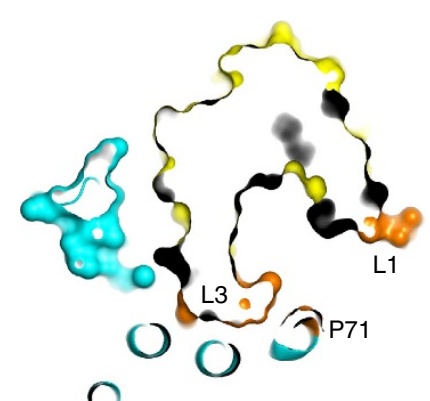

O b

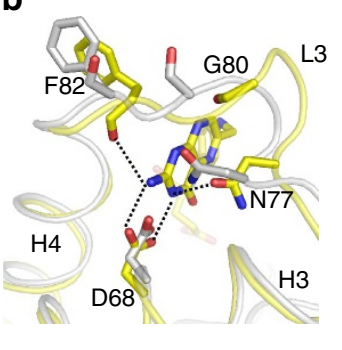

C

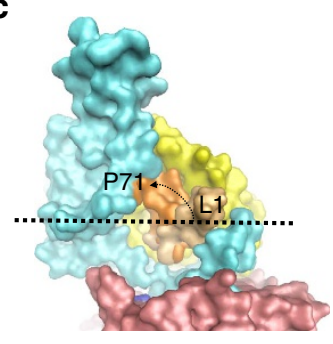

e

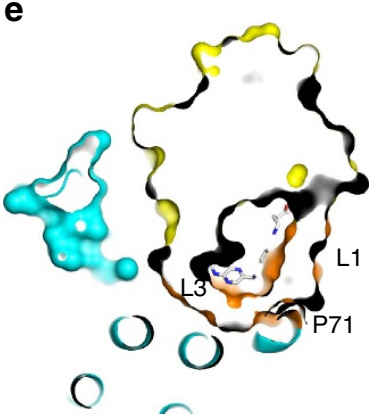

Figure 4 | Comparison of folate-bound FolT1 and substrate-free FolT2. (a) Structural alignment of folate-bound FolT1 (pale yellow) and substrate-free FolT2 (bright yellow). The viewpoint is from the extracytoplasmic side of the membrane. The carbon atoms of folate are shown in pale yellow and loops L1 and L3 are coloured in pale and bright orange for FolT1 and FolT2, respectively. (b) Repositioning of loop L3 of FolT2 (grey) compared with FolT1 (yellow) disrupts the interactions with folate, of which the carbon atoms are coloured yellow. Binding-site residues are indicated using the one-letter code for amino acids. (c) Surface representation showing how loop L1 (orange) of substrate-bound FolT1 would clash with P71 (orange) of EcfT when forming a complex with the ECF module. Loop L1 of FolT2 is shown in pale orange for comparison. The dashed arrow shows the movement of loop L1 between FolT2 and substrate-bound FolT1. (d) Slice-through representation of ECF-FolT2 at the level of the dashed line in c. FolT2 is shown in surface representation with loops L1 and L3 in orange, and P71 of EcfT in orange surface representation. (e) The same slice as shown in $\mathbf{d}$ but with substrate-bound FolT1 replacing FolT2, with the carbon atoms of folate shown in grey. Loops L1 and L3 would clash with P71.

kinetics, close to the diffusion limit. From the outward-occluded state of solitary FolT1 (orientation shown in Fig. 1), small movements of loops L1 and L3 would be sufficient to open access to the substrate-binding site to the extracellular side of the membrane. High-affinity folate binding from the outside has indeed been demonstrated in whole cell assays ${ }^{17}$. The small 

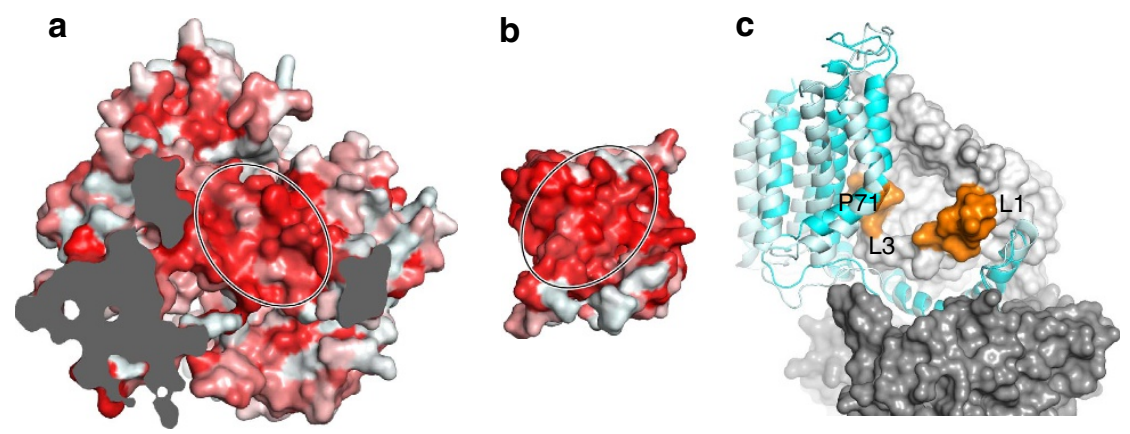

Figure 5 | Sites of interaction between EcfT and FolT2. (a) Slice-through of the ECF module in surface representation viewed from the extracellular side of the membrane. FolT2 has been deleted to show the hydrophobic platform on top of the coupling domain of EcfT, indicated by the oval. Colouring according to hydrophobicity, from red (hydrophobic) to grey (hydrophilic). (b) Surface of FolT2 interacting with the surface shown in a, using the same colour-coding. (c) Structural flexibility in the EcfT subunit. The apo and AMP-PNP-bound structures were superimposed by structural alignment of the ATPase subunits (dark grey surface representation). The resulting positions of the EcfT subunits are shown in cartoon representation with a viewpoint from the membrane plane. The coupling domains with coupling helices $\mathrm{CH} 1-3$ are in almost identical positions in the two complexes, but the membrane domains differ. The membrane domain in the apo-structure (grey) has hinged away compared with the AMP-PNP-bound structure (cyan). Proline 71 on TMH3 of EcfT as well as loops L1 and L3 in FolT2 are shown in orange.

conformational changes combined with high-affinity substrate binding make solitary S-component efficient scavengers for scarce and precious substrates in the environment of the bacteria.

Interaction of FolT with EcfT triggers substrate release. The high-affinity folate-binding site is destroyed in the structures of the ECF-FolT2 complex, which allows for accumulation of substrate in the cytoplasm, and likely results in higher off-rates than in the solitary S-components, leading to physiologically relevant transport rates. The free energy required to destroy the binding site must be provided by the interaction energy liberated upon docking of FolT to the ECF module. The coupling domain of EcfT provides the main surface for interaction with FolT2, with the three coupling helices forming a hydrophobic platform that binds an equally hydrophobic surface on helices 1 and 3 of FolT2 (Fig. 5a,b). Two alanines of the well-conserved AxxxA motif in helix 1 of FolT, which have been shown to be crucial for the interaction of other S-components with the ECF module ${ }^{11,15}$, are part of the interaction surface. The shapes of the two surfaces are highly complementary creating a tight fit.

The structure of the surface interacting with EcfT is identical in substrate-free FolT2 and folate-bound FolT1, which raises the question how the folate binding site is disrupted in the complex. The structures show that the association of FolT to EcfT via the hydrophobic interface allosterically disrupts the folate-binding site. The conformations of loops L1 and L3 in folate-bound FolT1 are incompatible with binding to the ECF module, because they would clash with TMH3 of the membrane domain of EcfT at the position of the conserved P71 (Fig. 4c-e). This proline also introduces a kink in $\mathrm{TMH} 3$, which directs the $\mathrm{N}$-terminal end of the helix away from the S-component, allowing a wide passageway from the folate-binding pocket to the cytoplasm (compare Figs $3 \mathrm{~b}$ and $4 \mathrm{c}-\mathrm{e}$ ). The steric clash with TMH3 shows that toppling of FolT can occur only if simultaneous displacement of loops L1 and L3 takes place, which results in loss of the folatebinding site. Thus, high-affinity binding of folate to FolT and interaction of EcfT with FolT are mutually exclusive.

The displacement of loops L1 and L3 in the toppled state is stabilized by interactions with EcfT. As will be discussed below, these interactions are probably much weaker than the interaction between the hydrophobic surfaces of FolT2 and the coupling domain of EcfT. The position of the L1 loop in the complexes is stabilized by a hydrogen bond between the backbone carbonyl group of G35 and the highly conserved R196 (the AMP-PNP structure) or N189 (in the apo structure) in the coupling domain of EcfT. The L3 loop interacts with a conserved serine- and threonine-rich stretch (ST $\phi \phi \mathrm{T} \phi \mathrm{TT}$, in which $\phi$ is a hydrophobic residue) in TMH4 of EcfT. The hydroxyl groups of the side chains of S123 and T127 from this motif form hydrogen bonds with the backbones of G76 and G80 in the L3 loop (Supplementary Fig. 7a), leading to a conformation of the L3 loop that cannot form the backbone interactions with the folate molecule found in FolT1, and forcing the side chain of N77 into the position of the pterin ring (Fig. 4b).

Besides loops L1 and L3, the C-terminal end of H5 from FolT2 also interacts with EcfT. The partial negative charge from the helix dipole binds to the positive charge of conserved R115 in TMH4 in the membrane domain of EcfT (Supplementary Fig. 7b). The interactions between EcfT and loops L1, L3 and $\mathrm{H} 5$ are not dependent on the amino-acid sequence of the S-component because either backbone groups (loops L1 and L3) or the negative charge from a helix dipole (H5) are involved. The sequence-independence of the interactions explains how S-components for different substrates can topple along and bind to the same ECF module.

AMP-PNP-binding does not lead to a closed conformation. Two patches of well-defined electron density were observed in EcfA and EcfA' of the complex crystallized in the presence of Mg-AMP-PNP and were assigned to the nucleotide molecules (Fig. 6). The two AMP-PNP molecules interact with conserved motifs that are essential for ATP binding and hydrolysis in other $\mathrm{ABC}$ transporters ${ }^{21}$. All the important motifs are present in both subunits, which share 35\% sequence identity (Supplementary Fig. 8). Even though AMP-PNP molecules are bound to both subunits, binding does not lead to the closed conformation of the ATPases. The AMP-PNP molecules interact only with motifs from a single ATPase subunit, and are separated from the signature motifs (LSGGQ) on the other subunit (Fig. 6c). Therefore the ATP hydrolysis sites are still incomplete. A detailed description of the AMP-PNP-binding sites is provided in the supplement.

Structural flexibility in the membrane domain of EcfT. Although the structures of the apo and AMP-PNP-bound complexes are very similar overall, the relative orientation of the 
a

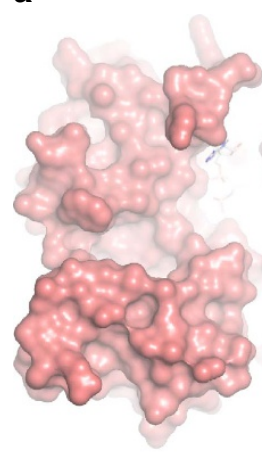

C
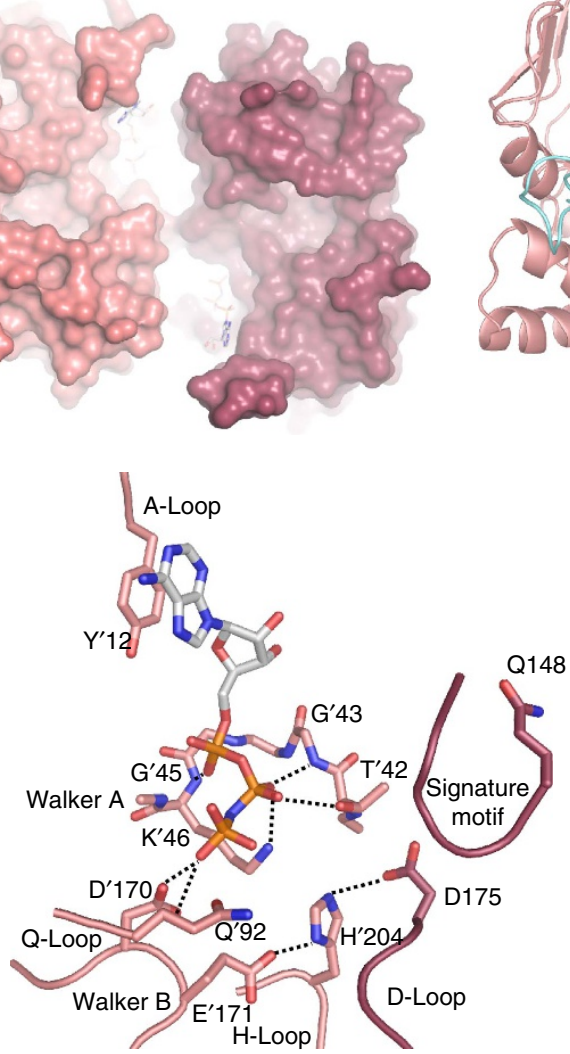

b

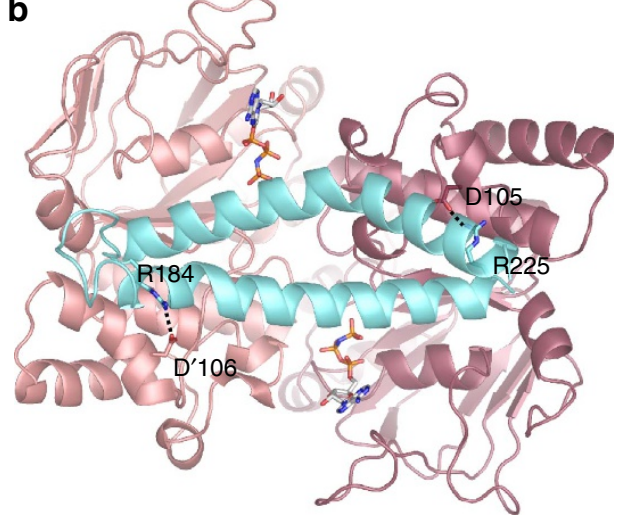

d

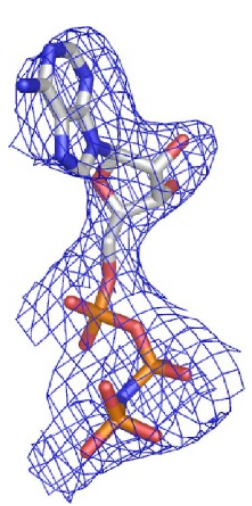

Figure 6 | AMP-PNP binding to the EcfAA' heterodimer. (a) Surface representation AMP-PNP-bound EcfAA' heterodimer coloured as in Fig. 3 with the AMP-PNP molecules in stick representation. The viewpoint is from the extracytoplasmic side (b) Cartoon representation of the AMP-PNP-bound EcfAA' heterodimer and the coupling helices $\mathrm{CH} 2$ and $\mathrm{CH} 3$ of EcfT. The conserved arginines at the $\mathrm{C}$-terminal end of the coupling helices, and the interacting aspartates in the EcfAA' heterodimer are shown in sticks. The interactions are indicated by the dashed lines. (c) AMP-PNP-binding site on EcfA' (coloured light red). The conserved motifs found in ABC transporter ATPases are indicated. (d) 2Fo-Fc electron density at $2 \sigma$ in blue contouring AMP-PNP (same viewpoint as in $\mathbf{c}$ ).

transmembrane and coupling domain of EcfT is different (Fig. 5c). The coupling domains of the two complexes are in identical positions relative to the two ATPases and FolT. Consequently, the hydrophobic interface that interacts with FolT (Fig. 5a) and the interface interacting with the ATPase subunits (Fig. 6b) are both intact in the two structures. In contrast, the transmembrane domains are in different orientations. In the AMP-PNP structure it is packed tightly against the FolT subunit and interacts with the loops L3 and the $\mathrm{C}$ terminus of $\mathrm{H} 5$, but in the apo structure it has hinged away and the two membrane proteins interact less tightly (Fig. 5c). It is likely that the structural flexibility of the membrane domain serves two functions. First, it allows toppling, which may require structurally different parts of FolT (loops L1, L3 and L5) to slide along the membrane part of EcfT. Second, EcfT has to be sufficiently flexible to allow the binding of different S-components with different sequences and structures in group II ECF transporters, which use the same ECF module ${ }^{15}$. The structural flexibility of the membrane domain of EcfT also suggests that the interactions with loop L3 and H5 from FolT are relatively weak, in contrast to the strong hydrophobic and van der Waals interactions between the rigid coupling domain of EcfT and FolT.

\section{Discussion}

Based on the work presented here and a wealth of data on various ECF transporters dating back to the 1970s, we tentatively provide a scheme for the transport cycle consistent with the available experimental data (Fig. 7). There are important differences compared with previously published models, which we will discuss below.

In all structures of complete ECF transporter complexes ${ }^{5,6,15}$, the S-components are trapped in an inward-facing posttranslocation state, in which they interact tightly with the coupling domain of EcfT via complementary hydrophobic surfaces. To escape from this state the interaction interface must be disrupted, which requires input of energy. Presumably the formation of a closed EcfA-EcfA' dimer by the binding of ATP, deforms the coupling helices from the EcfT subunit, thus destroying the hydrophobic interaction surface (step (1) in Fig. 7). ATP binding to the group II ECF-RibU transporter from Listeria monocytogenes ${ }^{8}$ has indeed been shown to drive the release of the S-component from the ECF module. The solitary S-component in the absence of substrate reorients to expose the empty binding site to the outside of the cell, ready to bind a new substrate (step (2) in Fig. 7). The high affinity of the S-components for their substrates subsequently allows for efficient scavenging from the environment (step (3) in Fig. 7). For group I ECF transporters, it has been suggested that the reorientation step does not lead to complete dissociation of the S-component from the ECF module ${ }^{16}$. Although it is possible that group I transporters indeed use a different mechanism, it is noteworthy that the experiments indicating the lack of dissociation were conducted in a nanodisc environment, which may prevent the detection of dissociation because of physical confinement. 


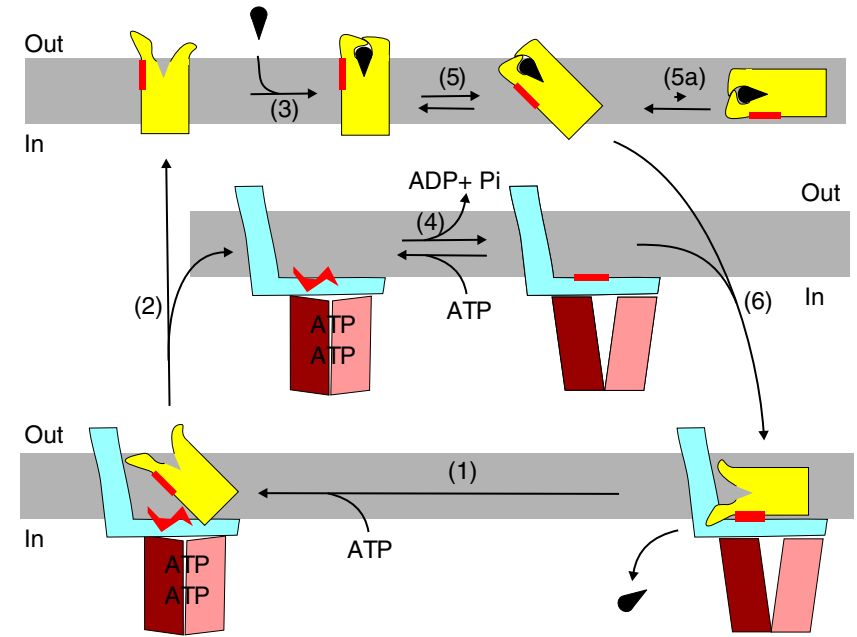

Figure 7 | Working model for the transport mechanism of group II ECF transporters. Colours of the subunits as in Fig. 3. Starting with the complex trapped in the post-translocation state, binding of ATP is needed to release the empty S-component by disruption of the hydrophobic interface (red) (step (1)). The S-component will reorient to the outward-facing state (2) and can bind substrate on the extracellular side of the membrane (3). ATP hydrolysis in the ECF module regenerates the binding platform for the S-component (4). Possibly futile ATP hydrolysis takes place in this stage. The substrate-bound S-component can topple over in the membrane possibly aided by the vicinity of the ECF module (5). The toppled S-component binds to the ECF module via the complementary hydrophobic surfaces, coloured in red (6). Binding of the S-component to the ECF module forces the disruption of the substrate-binding site and release of the substrate into the cytoplasm.

Our structural work shows that the binding of AMP-PNP to ECF-FolT2 is not sufficient to form the closed EcfA-EcfA' dimer. The lack of closure contrasts with the observed full closure when AMP-PNP binds to a partial complex of the ATPase subunits without the membrane subunits ${ }^{8}$. Apparently, closure is more difficult in the context of the complete complex. In other $\mathrm{ABC}$ transporters the binding of AMP-PNP sometimes led to closed ATPase subunits (for example, in the maltose transporter ${ }^{22,23}$ ), but in other cases left the ATPases in an open conformation ${ }^{24}$.

ATP is hydrolysed when the transport cycle proceeds. The requirement of full ATP hydrolysis for transport in lipid bilayers is obvious from the transport experiments presented in Fig. 2. The high basal ATPase activity of the ECF-FolT2 transporter when reconstituted in liposomes suggests that continuous ATP turnover may take place in the ECF module (step (4) in Fig. 7). Futile ATP consumption may be an acceptable trade-off to ensure capturing as much as possible of scarcely available nutrients. The high basal ATPase activity probably can only be sustained in the cell if low amounts of the ECF module are present. Indeed, the levels of the ECF module are usually much lower than the levels of S-components ${ }^{2}$.

In previously published models it has been postulated that toppling of the S-component from the outward- to the inwardfacing orientation requires strict coupling to ATP hydrolysis ${ }^{8,16}$. In contrast, we hypothesize that solitary substrate-loaded S-components have an intrinsic ability to topple over in the lipid bilayer (step (5) in Fig. 7). Such toppling may be possible because the substrate-bound S-components form compact structures without exposed charged residues on the extracellular face. The toppled proteins will be trapped by the hydrophobic binding platform of EcfT, which consequently destroys the highaffinity binding site and leads to substrate release in the cytoplasm (step (6) in Fig. 7). A strong indication that spontaneous toppling can indeed take place comes from the observation that some S-components can mediate substrate transport in the absence of an ECF module ${ }^{25-28}$.

Transport by solitary S-components is incompatible with strict coupling between ATP hydrolysis in the ECF module and toppling of the substrate-loaded S-component. Moreover, there is no conclusive experimental evidence for such coupling. For the group I transporter BioMNY, it has been shown that ATP hydrolysis is required for substrate release, but the data did not reveal the sequence of events ${ }^{16}$. ATP hydrolysis may take place in the absence of the S-component, only to reset the ECF module to the state that can interact tightly with the S-component again (steps (4) and (6) in Fig. 7). Even though strict coupling between ATP hydrolysis and toppling may not be necessary, it is possible that continuous ATP hydrolysis by the ECF module (step (4) in Fig. 7) facilitates toppling by distorting the lipid bilayer in the vicinity of the S-component.

In the 1970s, shared use of the ECF module by different S-components in group II transporters had already been proposed based on in vivo uptake experiments ${ }^{7}$. These experiments showed that substrate-loaded S-components compete more efficiently for the limited number of ECF modules than the substrate-free proteins. Recently these experiments have been reproduced using recombinant proteins

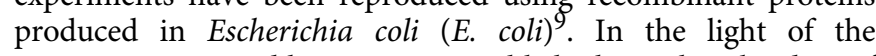
structures presented here it is very unlikely that tighter binding of substrate-loaded than substrate-free S-components to the ECF module would cause the competitive advantage. In fact, substratebound FolT seems to be unable to interact with the ECF module because of steric clashes between loops L1 and L3, and the membrane domain of EcfT. To explain the competition experiments, we hypothesize that substrate-loaded S-components do not bind more tightly to the ECF module than the substratefree proteins, but they can reach the binding platform more efficiently. The observed competition is then caused by differences in the kinetics of toppling. Substrate-free S-components cannot topple over as easily because their hydrophilic loops L1 and L3 are fully exposed. Therefore they are unable to reach the hydrophobic interaction platform of EcfT, even though they would bind to it very tightly if they could.

Importantly, the mechanism of competition described above does not require the existence of a stable state in which the ECF module is in complex with a substrate-loaded S-component. The existence of such a state was postulated when structures of the same S-component in the substrate-bound and apoconformation were not yet known ${ }^{8,16}$. In the work on the group I transporter ECF-BioY, the authors detected biotin binding to the solitary BioY, but not to the full complex. The authors postulate that an inaccessible (occluded) binding site exists in the complex ${ }^{16}$. Based on the structural data presented here and published previously, we explain the lack of binding by the disruption of the binding site, and consequent extrusion of biotin on the cytoplasmic side. The scheme presented here does not require the postulation of an occluded state, yet can explain the ensemble of experimental data, and therefore is more parsimonious than previous models.

For the group II transporter ECF-RibU the existence of a stable riboflavin-bound full complex has also been proposed. A mutant that could bind but not hydrolyse ATP readily exchanged its S-component RibU with a fluorescently labelled solitary RibU molecule in the presence of ATP in detergent solution ${ }^{8}$. Although this experiment elegantly supports the 'catch-and-release' mechanism of S-components, it does not require that the substrate riboflavin is bound to RibU in the complex. Even if riboflavin would bind to the full complex, the affinity might be 
much lower than for solitary RibU, consistent with the model proposed here.

The working model for the mechanism of transport presented in Fig. 7 accounts for the observed high basal ATPase activity, the competition of different S-components for the same ECF module, the release of S-component from the ECF module during turnover and substrate release from the S-component during toppling. This model will be used as handle for further experiments to study the toppling mechanism.

\section{Methods}

Cloning. The genes encoding EcfA, EcfA' and EcfT of L. delbrueckii subsp. bulgaricus are annotated as $c b i O$ (both for LDB_RS01805, ecfA and LDB_RS01810 $e c f A^{\prime}$ ) and $c b i Q$ (LDB_RS01815, ecfT). The ECF module operon was cloned downstream of the first arabinose inducible promoter of the p2BAD vector ${ }^{29}$ between the BspE1 and BglII sites, in-frame with a sequence coding for a $\mathrm{N}$-terminal His $10^{-}$-tag and TEV cleavage site before EcfA. folT1 (LDB_RS07025) or folT2 (LDB_RS07030) was cloned downstream of the second promoter, between the $\mathrm{XbaI}$ and $\mathrm{XhoI}$ sites in-frame with a sequence coding for a C-terminal STREPII tag (WSHPQFEK). The p2BAD His ${ }_{10}$-ECF-FolT1/2-STREPII plasmid was transformed into $\mathrm{Ca}^{2+}$-competent cells of the E. coli strain MC1061.

Solitary folT1 and folT2 were cloned in the pREnHis vector, which contains the sequence encoding for a $\mathrm{N}$-terminal $\mathrm{His}_{8}$-tag. Subsequently, the vectors were converted into $L$. lactis expression vectors using the vector backbone exchange protocol $^{30}$. The resulting pNZnHisFolT1/2 vectors were transformed into electrocompetent cells of the L. lactis strain NZ9000 ${ }^{31}$. Mutations in the FolT1/2 sequence were introduced using the primers given in Supplementary Table 1.

Expression and membrane vesicle preparation. Expression of ECF-FolT1/2 was performed in a 5-1 flask containing 21 of yeast trypton medium $\left(8 \mathrm{gl}^{-1}\right.$ Bacto trypton, $5 \mathrm{gl}^{-1}$ Bacto yeast extract, $2.5 \mathrm{gl}^{-1} \mathrm{NaCl}$ ), supplemented with $2.5 \mathrm{mM}$ potassium phosphate $(\mathrm{KPi}), \mathrm{pH} 7.0,0.5 \%$ glycerol and $100 \mathrm{\mu g} \mathrm{ml}^{-1}$ ampicillin. The E. coli MC1061 cells with p2BAD His 10 -ECF-FolT1/2-STREPII were grown at $37^{\circ} \mathrm{C}, 200$ r.p.m. to an $\mathrm{OD}_{600}$ of 0.8 , after which the temperature was reduced to $25^{\circ} \mathrm{C}$. After allowing the cultures to cool down for $20 \mathrm{~min}$, expression was induced by addition of $1.0 \times 10^{-2} \%$ arabinose. After $3 \mathrm{~h}$ of expression the cells were collected by centrifugation $\left(15 \mathrm{~min}, 7,446 g, 4^{\circ} \mathrm{C}\right)$, washed in buffer A $(50 \mathrm{mM} \mathrm{KPi}, \mathrm{pH}$ $7.5)$ and resuspended in the buffer B (50 mM KPi, pH 7.5, 10\% glycerol). Membrane vesicles were either prepared immediately, or the resuspended cells were stored at $-80^{\circ} \mathrm{C}$ after flash freezing in liquid nitrogen.

For folate-binding assays substrate-free FolT1 and FolT2 were produced in L. lactis cells grown semi-anaerobically in chemically defined medium ${ }^{32}$ without folate, and supplemented with $2.0 \%\left(\mathrm{w} / \mathrm{v}\right.$ ) glucose, $5 \mu \mathrm{g} \mathrm{ml}^{-1}$ chloramphenicol, in a $2 \mathrm{l}$ fermenter at $30^{\circ} \mathrm{C}$ and $\mathrm{pH} 6.5$. The cell culture was induced at an $\mathrm{OD}_{600}$ of $\sim 1.5$ by addition of $0.1 \%(\mathrm{v} / \mathrm{v})$ culture supernatant of a Nisin A-producing $\operatorname{strain}^{31}$. For crystallization FolT1 and FolT2 were produced in L. lactis cells grown in M17 broth (Difco), supplemented with 2.0\% (w/v) glucose, $5 \mu \mathrm{g} \mathrm{ml}^{-1}$ chloramphenicol, in a 11 bottle. In this case the cell culture was induced at $\mathrm{OD}_{600}$ $\sim 0.8$ by addition of $0.2 \%(\mathrm{v} / \mathrm{v})$ culture supernatant of the Nisin A-producing strain. After $3 \mathrm{~h}$ of expression, the cell cultures were collected and washed as described above for the expression of ECF-FolT2, but in $50 \mathrm{mM} \mathrm{KPi} \mathrm{pH7.0} \mathrm{and}$ $10 \%$ glycerol.

Before membrane vesicle preparation, $1 \mathrm{mM} \mathrm{MgSO}_{4}$ and $\sim 50-100 \mu \mathrm{g} \mathrm{ml}^{-1}$ DNase were added to the cells. The cells were lysed by high-pressure disruption (Constant Cell Disruption System Ltd, UK, two passages at $25 \mathrm{kPsi}$ for E. coli cells or at $39 \mathrm{kPsi}$ for $\mathrm{L}$. lactis cells, $5^{\circ} \mathrm{C}$ ) and cell debris was removed by low-speed centrifugation (30 min, $12,074 g, 4^{\circ} \mathrm{C}$ ). Membrane vesicles were collected by ultracentrifugation ( $120 \mathrm{~min}, 193,727 \mathrm{~g}, 4^{\circ} \mathrm{C}$ ), and resuspended in buffer B to a final volume of $5 \mathrm{ml}$ per 11 of cell culture. Subsequently, the membrane vesicles were aliquoted, flash frozen in liquid nitrogen and stored at $-80^{\circ} \mathrm{C}$. The total protein concentration in the membrane vesicles was determined by Bradford Protein Assay (Bio-Rad).

Protein purification. For the purification of ECF-FolT2, membrane vesicles were thawed rapidly and solubilized in buffer C (50 mM KPi, pH 7.5, $300 \mathrm{mM} \mathrm{NaCl}$, $15 \mathrm{mM}$ imidazole, $1 \%$ (w/v) $n$-dodecyl- $\beta$-D-maltopyranoside (DDM, Anatrace)) for $1 \mathrm{~h}$ at $4{ }^{\circ} \mathrm{C}$, while gently rocking. Unsolubilized material was removed by centrifugation $\left(20 \mathrm{~min}, 442,907 \mathrm{~g}, 4^{\circ} \mathrm{C}\right)$. The supernatant was incubated for $1 \mathrm{~h}$ at $4{ }^{\circ} \mathrm{C}$ under gently rocking with $\mathrm{Ni}^{2+}$-sepharose resin (column volume of $0.5 \mathrm{ml}$ ), which had been equilibrated with buffer D (50 mM KPi, pH 7.5, $300 \mathrm{mM} \mathrm{NaCl}, 50 \mathrm{mM}$ imidazole, $0.05 \%(\mathrm{w} / \mathrm{v}) \mathrm{DDM})$. Subsequently, the suspension was poured into a 10-ml disposable column (Bio-Rad) and the flow through was collected. The column material was washed with $15 \mathrm{ml}$ of buffer D. The ECF-FolT2 complex was eluted in three fractions of buffer E (50 mM KPi, pH 7.5, $300 \mathrm{mM} \mathrm{NaCl}, 500 \mathrm{mM}$ imidazole, $0.05 \%(\mathrm{w} / \mathrm{v}) \mathrm{DDM})$ of 400,750 and $500 \mu \mathrm{l}$, respectively. An amount of $1 \mathrm{mM}$ of EDTA was added to the second elution fraction to remove co-eluted $\mathrm{Ni}^{2}+$ ions. Subsequently, the second elution fraction was purified by size-exclusion chromatography using a Superdex 200 10/300 gel filtration column (GE
Healthcare), equilibrated with buffer F (50 mM KPi, pH 7.5, $150 \mathrm{mM} \mathrm{NaCl}, 0.05 \%$ (w/v) DDM) in case of purification for reconstitution or equilibrated with buffer $\mathrm{G}$ (20 mM Tris, $\mathrm{pH} 8.0,150 \mathrm{mM} \mathrm{NaCl}, 0.05 \%(\mathrm{w} / \mathrm{v}) \mathrm{DDM}$ ) in case of purification for crystallization. After size-exclusion chromatography, the fractions containing the ECF-FolT2 complex were combined and used directly for reconstitution, or concentrated by the use of a Vivaspin 500 concentrating device with a molecular weight cutoff of $100 \mathrm{kDa}$ (Sartorius stedim) to a final concentration of $5-8 \mathrm{mg} \mathrm{ml}^{-1}$ when used for crystallization.

FolT1 and FolT2 were purified using the same protocol, but with different buffers. Solubilization of the membrane vesicles was performed in buffer $\mathrm{H}(50 \mathrm{mM}$ $\mathrm{KPi}, \mathrm{pH} 7.0,200 \mathrm{mM} \mathrm{KCl}, 1 \%(\mathrm{w} / \mathrm{v}) \mathrm{DDM})$, the $\mathrm{Ni}^{2+}$-sepharose resin was equilibrated and washed with buffer I (50 mM KPi, pH 7.0, $200 \mathrm{mM} \mathrm{KCl}, 50 \mathrm{mM}$ imidazole, $0.35 \%(\mathrm{w} / \mathrm{v}) n$-nonyl- $\beta$-D-glucopyranoside (NG, Anatrace)), the proteins were eluted from the $\mathrm{Ni}^{2+}$-sepharose column using buffer J (50 mM KPi, $\mathrm{pH} 7.0$, $200 \mathrm{mM} \mathrm{KCl}, 500 \mathrm{mM}$ imidazole, $0.35 \%$ (w/v) NG) and the Superdex $20010 / 300$ gel filtration column was equilibrated with buffer K (50 mM KPi, pH 7.0, $150 \mathrm{mM}$ $\mathrm{KCl}, 0.35 \%(\mathrm{w} / \mathrm{v}) \mathrm{NG})$. After size-exclusion chromatography, the fractions containing FolT1 or FolT2 were combined and directly used for substrate-binding assays. When FolT1 was used for crystallization, the protein was incubated in the presence of $100 \mu \mathrm{M}$ folate overnight at $4{ }^{\circ} \mathrm{C}$ under gently rocking and subsequently concentrated by the use of a Vivaspin 500 concentrating device with a molecular weight cutoff of $50 \mathrm{kDa}$ (Sartorius stedim) to a final concentration of $8 \mathrm{mg} \mathrm{ml}^{-1}$

Crystallization and structure determination. Initial crystals of the apo ECFFolT2 complex were found in the G8 condition $(50 \mathrm{mM}$ Tris, pH 7.5, $17 \%(\mathrm{v} / \mathrm{v})$ PEG350 MME) of the MemGold2 HT-96 screen (Molecular Dimensions) using a Mosquito robot (TTP Labtech, UK) and diffracted up to $8 \AA$ resolution. Optimizations using the Additive (HR2-428) and Detergent (HR2-408) screens of Hampton Research led to an optimized condition of $50 \mathrm{mM}$ Tris, $\mathrm{pH} 7.5,17 \%(\mathrm{v} / \mathrm{v})$ PEG350 MME, $10 \mathrm{mM}$ spermidine, $2 \%(\mathrm{w} / \mathrm{v}) \mathrm{NG}$, which yielded crystals diffracting up to $3.7 \AA$ A resolution. Multiple rounds of seeding in 24-well hanging-drop crystallization plates resulted in diamond-shaped crystals, which grew in clumps and diffracted up to $2.7 \AA$ resolution. Using the apo ECF-FolT2 crystals for seeding, ECF-FolT2 in the presence of $10 \mathrm{mM} \mathrm{MgCl} 2,10 \mathrm{mM}$ AMP-PNP and $1 \mu \mathrm{M}$ folate crystallized in single diamond-shaped crystals diffracting up to $3.0 \AA$ A resolution. Diffraction data were collected at the European Synchrotron Radiation Facility (ESRF) at beamline ID23-1, Grenoble ( $\lambda=0.97 \AA, T=100 \mathrm{~K}$, ID23). The crystals of apo ECF-FolT2 belong to space group P1 (unit cell parameters: $a=88.82 \AA$, $b=95.32 \AA, c=107.57 \AA, \alpha=83.45^{\circ}, \beta=65.75^{\circ}$ and $\left.\gamma=61.99^{\circ}\right)$. The crystals of ECF-FolT2 grown in the presence of $10 \mathrm{mM} \mathrm{MgCl}_{2}, 10 \mathrm{mM}$ AMP-PNP and $1 \mu \mathrm{M}$ folate belong to space group P1 (unit cell parameters: $a=90.07 \AA, b=97.26 \AA$, $c=105.45 \AA, \alpha=84.67^{\circ}, \beta=64.78^{\circ}$ and $\left.\gamma=62.59^{\circ}\right)$. Crystals of FolT1 were obtained using the hanging-drop vapour-diffusion technique using a reservoir solution of $100 \mathrm{mM}$ Tris, $\mathrm{pH} 7.5,24 \%$ (v/v) PEG350 MME. These crystals had a rectangular shape and diffracted up to $2.8 \AA$ resolution. Diffraction data were collected at the Swiss Light Source (SLS), Villigen $(\lambda=0.92 \AA, T=100 \mathrm{~K}, \mathrm{X} 06 \mathrm{SA})$. The FolT1 crystals belong to space group C 121 (unit cell parameters: $a=108.91 \AA, b=77.54 \AA, c=89.45 \AA, \alpha=90.00^{\circ}, \beta=116.36^{\circ}$ and $\left.\gamma=90.00^{\circ}\right)$. All data sets suffered from severe anisotropy and were treated with the diffraction anisotropy server ${ }^{33}$. Data were processed with XDS and molecular replacement was carried out with Phaser $M^{34}$. For the apo structure, molecular replacement with the EcfA-EcfA' heterodimer structure of Thermotoga maritima (PDB ID: 4HLU) yielded a solution for the EcfA and EcfA' subunit, in which the structures of EcfT and FolT2 were built in by hand with Coot ${ }^{35}$, guided by the structure of ECF-FolT of $L$. brevis (PDB ID: 4HUQ), and with the help of Phenix autobuild ${ }^{36}$, using the AMP-PNP bound structure. To override bias problems Rosetta-based $\mathrm{MR}^{37}$ was also used and refinement was performed with Phenix refinement ${ }^{38}$. The

Ramachandran statistics are $91.9 \%, 90.3 \%$ and $96.1 \%$ for favoured regions for apo ECF FolT2, AMP-PNP-bound ECF FolT2 and folate-bound FolT, respectively, and $7.4 \%, 8.8 \%$ and $3.9 \%$ for allowed regions. The statistics for data collection and refinement are summarized in Table 1 . A stereo view of $2 \mathrm{Fo}-\mathrm{Fc}$ electron density is shown in Supplementary Fig. 9.

Substrate-binding assay by intrinsic fluorescence titration. The affinity of FolT1, FolT2 and their mutants for folate was determined by performing an intrinsic fluorescence titration assay at $25^{\circ} \mathrm{C}$ (refs 39,40), using a Spec Fluorlog 322 fluorescence spectrophotomer. The protein was diluted in buffer $\mathrm{K}$ to a final concentration of $50 \mathrm{nM}$ (final volume of $800 \mu \mathrm{l}$ ) and added to a 1,000- $\mu \mathrm{l}$ quartz cuvette. After incubation for $5 \mathrm{~min}$, folate was added in $1 \mu \mathrm{l}$ steps using a Harvard apparatus syringe pump equipped with a $100-\mu$ l gastight glass syringe (Hamilton). Using an excitation wavelength of $280 \mathrm{~nm}$, the emission was recorded at $350 \mathrm{~nm}$. After each substrate addition step, $10 \mathrm{~s}$ were allowed for mixing and the fluorescence signals were averaged over a time range of $15 \mathrm{~s}$. Data analysis was performed as described previously ${ }^{39}$.

Reconstitution into proteoliposomes. Purified ECF-FolT2 was reconstituted in proteoliposomes as described previously ${ }^{41}$, using a protein to lipid (w/w) ratio of 1:250 and using liposomes composed of E. coli polar lipids and egg phosphatidylcholine $(3: 1(\mathrm{w} / \mathrm{w})$ ratio). 
Radiolabelled folate transport assay. To use in the transport assay or the ATPase activity assay, proteoliposomes were thawed and $5 \mathrm{mM}$ of $\mathrm{Na}_{2}$-ATP plus $5 \mathrm{mM}$ of $\mathrm{MgSO}_{4}, 5 \mathrm{mM} \mathrm{Na}_{2}$-ADP plus $5 \mathrm{mM} \mathrm{MgSO}_{4}, 5 \mathrm{mM} \mathrm{Na}_{2}$-ATP plus $5 \mathrm{mM}$ EDTA or $5 \mathrm{mM}$ AMP-PNP plus $5 \mathrm{mM} \mathrm{MgSO}$ were added. To include these compounds in the proteoliposomes, three cycles of flash-freezing in liquid nitrogen and quick thawing of the proteoliposomes were performed. Subsequently, the proteoliposomes were extruded through a $400-\mathrm{nm}$ pore size polycarbonate filter (Avestin, 11 passages). To wash away the external nucleotides, the proteoliposomes were diluted 32 times to a final volume of $8 \mathrm{ml}$. After centrifugation ( $45 \mathrm{~min}$, $285,775 \mathrm{~g}, 4^{\circ} \mathrm{C}$ ), the proteoliposomes were resuspended in buffer $\mathrm{A}$ to a final concentration of $0.5-1 \mu \mathrm{g}$ ECF-FolT2 $\mu^{-1}$. For each time point in the transport assays, a reaction volume of $200 \mu \mathrm{l}$ of buffer A supplemented with $5 \mathrm{nM}$ of $[3,5,7$, 9- ${ }^{3} \mathrm{H}$ ] radiolabelled folate and $95 \mathrm{nM}$ of non-radiolabelled folate was incubated at $25^{\circ} \mathrm{C}$ while being stirred. Transport was started by adding $1 \mu \mathrm{g}$ of ECF-FolT2, reconstituted in proteoliposomes. At the indicated time point, $2 \mathrm{ml}$ of stop buffer (ice-cold buffer A) was added and the reaction was rapidly filtered over a BA-85 nitrocellulose filter. After washing the filter with another $2 \mathrm{ml}$ of stop buffer, the filter was dried for $1 \mathrm{~h}$ at $80^{\circ} \mathrm{C}$. Subsequently, the filter was dissolved in $5 \mathrm{ml}$ of Filter Count scintillation liquid (PerkinElmer) and the levels of radioactivity were determined using a PerkinElmer Tri-Carb 2800 TR isotope counter. For the efflux experiments, uptake of radiolabelled folate by $\mathrm{MgATP}$-loaded proteoliposomes was allowed for $16 \mathrm{~min}$, after which $5 \mathrm{mM}$ of MgATP, $5 \mathrm{mM}$ MgADP, $5 \mathrm{mM}$ ATP plus $5 \mathrm{mM}$ EDTA or $100 \mu \mathrm{M}$ non-radiolabelled folate was added and efflux was followed for an additional $8 \mathrm{~min}$.

ATPase activity assay. The ATPase activity of ECF-FolT2 reconstituted in proteoliposomes (loaded with 0 or $100 \mathrm{nM}$ of folate as described above) was measured by using a coupled enzyme assay, in which the amount of ADP produced (and thus the amount of ATP hydrolysed) is coupled stoichiometric with the oxidation of $\mathrm{NADH}^{42}$. The assay was performed at $30^{\circ} \mathrm{C}$ in a 96 -well plate and the absorbance at $340 \mathrm{~nm}$ was measured by a Synergy MX-96-well plate reader (BioTek Instruments, Inc.). A volume of $200 \mu \mathrm{l}$ of reaction solution per well contained $50 \mathrm{mM} \mathrm{KPi}, \mathrm{pH} 7.5,200 \mathrm{mM} \mathrm{NaCl}, 5.2 \mathrm{nM}(1.2 \mu \mathrm{g})$ of ECF-FolT2, $4 \mathrm{mM}$ sodium phosphoenolpyruvate, $0.3 \mathrm{mM} \mathrm{NADH}$ and $3.5 \mu \mathrm{l}$ of pyruvate kinase/lactic dehydrogenase enzyme mixture from rabbit muscle (Sigma-Aldrich) in 50\% glycerol. The reaction solutions were supplemented with 0 or $100 \mathrm{nM}$ folate as indicated (folate was thus present both on the inside and the outside of the proteoliposomes in the $100 \mathrm{nM}$ folate condition). After incubation of the reaction solutions for $3 \mathrm{~min}$ at $30^{\circ} \mathrm{C}, 1 \mathrm{mM}$ of MgATP, $\mathrm{pH} 7.5$, was added to each of the reactions and the absorbance of NADH at $340 \mathrm{~nm}$ was followed for $7 \mathrm{~min}$. The ATPase activity was expressed in $\mu \mathrm{mol}$ of ATP hydrolysed per min per mg of ECF-FolT2).

\section{References}

1. Rodionov, D. A., Hebbeln, P., Gelfand, M. S. \& Eitinger, T. Comparative and functional genomic analysis of prokaryotic nickel and cobalt uptake transporters: evidence for a novel group of ATP-binding cassette transporters. J. Bacteriol. 188, 317-327 (2006).

2. Rodionov, D. A. et al. A novel class of modular transporters for vitamins in prokaryotes. J. Bacteriol. 191, 42-51 (2009).

3. Eitinger, T., Rodionov, D. A., Grote, M. \& Schneider, E. Canonical and ECF-type ATP-binding cassette importers in prokaryotes: diversity in modular organization and cellular functions. FEMS Microbiol. Rev. 35, 3-67 (2011).

4. Slotboom, D. J. Structural and mechanistic insights into prokaryotic energy-coupling factor transporters. Nat. Rev. Microbiol. 12, 79-87 (2014).

5. $\mathrm{Xu}, \mathrm{K}$. et al. Crystal structure of a folate energy-coupling factor transporter from Lactobacillus brevis. Nature 497, 268-271 (2013).

6. Wang, T. et al. Structure of a bacterial energy-coupling factor transporter. Nature 497, 272-276 (2013).

7. Henderson, G. B., Zevely, E. M. \& Huennekens, F. M. Mechanism of folate transport in Lactobacillus casei: evidence for a comonent shared with the thiamine and biotin transport systems. J. Bacteriol. 137, 1308-1314 (1979).

8. Karpowich, N. K., Song, J. M., Cocco, N. \& Wang, D.-N. ATP binding drives substrate capture in an ECF transporter by a release-and-catch mechanism. Nat. Struct. Mol. Biol. 22, 565-571 (2015).

9. Majsnerowska, M., Beek, J., Stanek, W. K., Duurkens, R. H. \& Slotboom, D. J. Competition between different S-components for the shared energy coupling factor module in energy coupling factor transporters. Biochemistry 54, 4763-4766 (2015).

10. Zhang, P., Wang, J. \& Shi, Y. Structure and mechanism of the S component of a bacterial ECF transporter. Nature 468, 717-720 (2010).

11. Erkens, G. B. et al. The structural basis of modularity in ECF-type ABC transporters. Nat. Struct. Mol. Biol. 18, 755-760 (2011).

12. Berntsson, R. P. et al. Structural divergence of paralogous $S$ components from ECF-type ABC transporters. Proc. Natl Acad. Sci. USA 109, 13990-13995 (2012).

13. $\mathrm{Yu}$, Y. et al. Planar substrate-binding site dictates the specificity of ECF-type nickel/cobalt transporters. Cell Res. 24, 267-277 (2014).
14. Zhao, Q. et al. Structures of FolT in substrate-bound and substrate-released conformations reveal a gating mechanism for ECF transporters. Nat. Commun. 6, 7661 (2015).

15. Zhang, M. et al. Structure of a pantothenate transporter and implications for ECF module sharing and energy coupling of group II ECF transporters. Proc. Natl. Acad. Sci. USA 111, 18560-18565 (2014).

16. Finkenwirth, F. et al. ATP-dependent conformational changes trigger substrate capture and release by an ECF-type biotin transporter. J. Biol. Chem. 290, 16929-16942 (2015).

17. Eudes, A. et al. Identification of genes encoding the folate- and thiamine-binding membrane proteins in Firmicutes. J. Bacteriol. 190, 7591-7594 (2008).

18. von Heijne, G. Membrane-protein topology. Nat. Rev. Mol. Cell Biol. 7, 909-918 (2006).

19. Majsnerowska, M. et al. Substrate-induced conformational changes in the S-component ThiT from an energy coupling factor transporter. Structure 21, 861-867 (2013).

20. ter Beek, J., Duurkens, R. H., Erkens, G. B. \& Slotboom, D. J. Quaternary structure and functional unit of energy coupling factor (ECF)-type transporters. J. Biol. Chem. 286, 5471-5475 (2011).

21. ter Beek, J., Guskov, A. \& Slotboom, D. J. Structural diversity of ABC transporters. J. Gen. Physiol. 143, 419-435 (2014).

22. Oldham, M. L. \& Chen, J. Snapshots of the maltose transporter during ATP hydrolysis. Proc. Natl Acad. Sci. USA 108, 15152-15156 (2011).

23. Oldham, M. L. \& Chen, J. Crystal structure of the maltose transporter in a pretranslocation intermediate state. Science 332, 1202-1205 (2011).

24. Hohl, M., Briand, C., Grütter, M. G. \& Seeger, M.A. Crystal structure of a heterodimeric ABC transporter in its inward-facing conformation. Nat. Struct. Mol. Biol. 19, 395-402 (2012).

25. Hebbeln, P., Rodionov, D. a, Alfandega, A. \& Eitinger, T. Biotin uptake in prokaryotes by solute transporters with an optional ATP-binding cassettecontaining module. Proc. Natl Acad. Sci. USA 104, 2909-2914 (2007).

26. Finkenwirth, F., Kirsch, F. \& Eitinger, T. Solitary BioY proteins mediate biotin transport into recombinant Escherichia coli. J. Bacteriol. 195, 4105-4111 (2013).

27. Finkenwirth, F., Kirsch, F. \& Eitinger, T. A versatile Escherichia coli strain for identification of biotin transporters and for biotin quantification. Bioengineered 5, 129-132 (2014).

28. Kirsch, F. \& Eitinger, T. Transport of nickel and cobalt ions into bacterial cells by S components of ECF transporters. Biometals 27, 653-660 (2014).

29. Birkner, J. P. \& Poolman, B. Hydrophobic gating of mechanosensitive channel of large conductance evidenced by single-subunit resolution. Proc. Natl Acad. Sci. USA 109, 12944-12949 (2012).

30. Geertsma, E. R. \& Poolman, B. High-throughput cloning and expression in recalcitrant bacteria. Nat. Methods 4, 705-707 (2007).

31. Kuipers, O. P., de Ruyter, P. G. G., Kleerebezem, M. \& de Vos, W. M. Quorum sensing-controlled gene expression in lactic acid bacteria. J. Biotechnol. 64, 15-21 (1998).

32. Berntsson, R. P. et al. Selenomethionine incorporation in proteins expressed in Lactococcus lactis. Protein Sci. 18, 1121-1127 (2009).

33. Strong, M. et al. Toward the structural genomics of complexes: crystal structure of a PE/PPE protein complex from Mycobacterium tuberculosis. Proc. Natl Acad. Sci. USA 103, 8060-8065 (2006).

34. McCoy, A. J. et al. Phaser crystallographic software. J. Appl. Crystallogr. 40, 658-674 (2007).

35. Emsley, P., Lohkamp, B., Scott, W. G. \& Cowtan, K. Features and development of Coot. Acta Crystallogr. D Biol. Crystallogr. 66, 486-501 (2010).

36. Terwilliger, T. C. et al. Iterative model building, structure refinement and density modification with the PHENIX AutoBuild wizard. Acta Crystallogr. D. Biol. Crystallogr. 64, 61-69 (2008).

37. DiMaio, F. et al. Improved molecular replacement by density- and energy-guided protein structure optimization. Nature 473, 540-543 (2011).

38. Adams, P. D. et al. PHENIX: a comprehensive Python-based system for macromolecular structure solution. Acta Crystallogr. D Biol. Crystallogr. 66, 213-221 (2010).

39. Erkens, G. B. \& Slotboom, D. J. Biochemical characterization of ThiT from Lactococcus lactis: a thiamin transporter with picomolar substrate binding affinity. Biochemistry 49, 3203-3212 (2010).

40. Swier, L. J. Y. M. et al. Structure-based design of potent small-molecule binders to the S-component of the ECF transporter for thiamine. Chembiochem 16, 819-826 (2015).

41. Geertsma, E. R., Nik Mahmood, N. a B., Schuurman-Wolters, G. K. \& Poolman, B. Membrane reconstitution of $\mathrm{ABC}$ transporters and assays of translocator function. Nat. Protoc. 3, 256-266 (2008).

42. Karasawa, A. et al. Physicochemical factors controlling the activity and energy coupling of an ionic strength-gated ATP-binding cassette $(\mathrm{ABC})$ transporter. J. Biol. Chem. 288, 29862-29871 (2013). 


\section{Acknowledgements}

We thank Josy ter Beek and Raj Singh for constructing the wild-type expression plasmids, Marysia Majsnerowska and Weronika Stanek for helpful suggestions for the transport experiments and Bert Poolman for critically reading the manuscript. This work was funded by the Netherlands Organization for Scientific Research (NWO; NWO ECHO grant 711.011.001 and NWO Vici grant 865.11.001 to D.J. Slotboom) and the European Research Council (ERC; ERC Starting Grant 282083 to D.J. Slotboom).

\section{Author contributions}

All authors contributed to all stages of the work.

\section{Additional information}

Accession codes. Atomic coordinates and structure factors for the crystal structures of folate-bound FolT1, AMP-PNP-bound ECF-FolT2 and apo ECF-FolT2 from L. delbrueckii have been deposited in the Protein Data Bank under the accession codes 5D0Y, 5D3M and 5D7T, respectively.
Supplementary Information accompanies this paper at http://www.nature.com/ naturecommunications

Competing financial interests: The authors declare no competing financial interests.

Reprints and permission information is available online at http://npg.nature.com/ reprintsandpermissions/

How to cite this article: Swier, L. J. Y. M. et al. Structural insight in the toppling mechanism of an energy-coupling factor transporter. Nat. Commun. 7:11072 doi: $10.1038 /$ ncomms11072 (2016).

(c) (i) This work is licensed under a Creative Commons Attribution 4.0 International License. The images or other third party material in this article are included in the article's Creative Commons license, unless indicated otherwise in the credit line; if the material is not included under the Creative Commons license, users will need to obtain permission from the license holder to reproduce the material. To view a copy of this license, visit http://creativecommons.org/licenses/by/4.0/ 